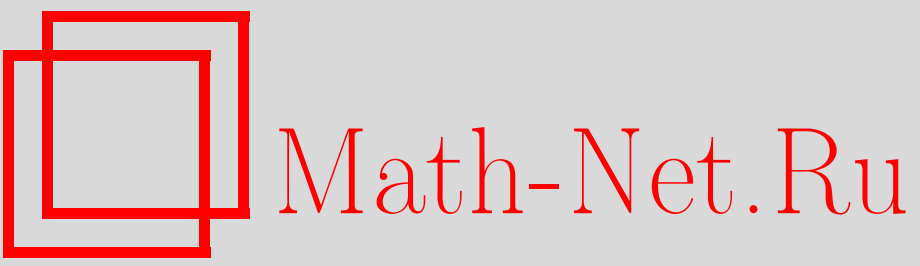

С. В. Людковский, Гауссовы меры на пространствах свободных петель, УМH, 2001, том 56, выпуск 5, 183-184

DOI: https://doi.org/10.4213/rm444

Использование Общероссийского математического портала Math-Net.Ru подразумевает, что вы прочитали и согласны с пользовательским соглашением

http://www.mathnet.ru/rus/agreement

Параметры загрузки:

IP : 18.208 .226 .222

26 апреля 2023 г., 15:17:54 


\title{
ГАУССОВЫ МЕРЫ НА ПРОСТРАНСТВАХ СВОБОДНЫХ ПЕТЕЛЬ
}

\author{
С. В. Людковский
}

Пространства свободных петель важны в дифференциальной геометрии, алгебраической топологии и теоретической физике. Данная работа посвящена построению квазиинвариантных гауссовых мер на пространствах $Y^{\xi}\left(M, s_{0} ; N, y_{0}\right)$ свободных петель $f: M \rightarrow N$ с $f\left(s_{0}\right)=y_{0}$ для многообразий $M$ и $N$, где $s_{0} \in M, y_{0} \in N$ - отмеченные точки.

1. Определения и обозначения. Каноническое замкнутое подмножество $Q$ в $X=\mathbb{R}^{n}$ или в $X=l_{2}$ над $\mathbb{R}$ назьвается квадрантом, если $Q:=\left\{x \in X: q_{j}(x) \geqslant 0, j \in \Lambda_{Q}\right\}$, где $q_{j} \in X^{*}$, $\Lambda_{Q} \subset \mathbb{N}$. Тогда аналогично [1] задаются $M$ и $N$ - сепарабельные римановы ориентированные метризуемые связные многообразия с углами, $\left(U_{l}, u_{l}, Q_{l}\right)$ и $\left(V_{i}, \psi_{i}, S_{i}\right)$ - карты атласов $M$ и $N$, где $u_{l}: U_{l} \rightarrow u_{l}\left(U_{l}\right) \subset Q_{l}, i \in \Lambda_{N} \subset \mathbb{N}$, причем пусть (i) $M$ является объединением двух канонических замкнутых подмногообразий $A_{1}$ и $A_{2}$ с углами, $A_{1} \cap A_{2}=\partial A_{1} \cap \partial A_{2}=: A_{3}$ и $\operatorname{codim} A_{3}=1$; (ii) $s_{0} \in A_{3}$; (iii) $A_{1}$ и $A_{2}$ являются $C^{0}\left([0,1] \times A_{j}, A_{j}\right)$-стягиваемыми в $s_{0}$, где $j \in\{1,2\}$; (iv) если $M$ бесконечномерно, то имеется последовательность вложенных подмногообразий $\eta_{m}^{m+1}: M_{m} \hookrightarrow M_{m+1} \quad \forall m \in \mathbb{N}$, удовлетворяющих (i)-(iii), а $s_{0, m}$ в $M_{m}$ соответствует $s_{0, m+1}=\eta_{m}^{m+1}\left(s_{0, m}\right)$ в $M_{m+1}, \operatorname{dim} M_{m}=n(m), 0<n(m)<n(m+1)$, $\bigcup_{m} M_{m}$ плотно в $M,\left.u_{i} \circ u_{j}^{-1}\right|_{u_{j}\left(U_{i} \cap U_{j}\right)} \rightarrow l_{2}$ имеют вид: $u_{i} \circ u_{j}^{-1}\left(\left(x^{l}: l \in \mathbb{N}\right)\right)=$ $\left(\alpha_{i, j, m}\left(x^{1}, \ldots, x^{n(m)}\right), \gamma_{i, j, m}\left(x^{l}: l>n(m)\right)\right)$, когда $\partial M=\varnothing$, а для любой компоненты границы $M_{0}$ в $M$ и $U_{j} \cap M_{0} \neq \varnothing$ имеем отображение $\varphi_{j}: U_{j} \cap M_{0} \rightarrow H_{l, Q}$, причем $\partial M_{m} \subset \partial M$ $\forall m$, где $H_{l, Q}:=\left\{x \in Q_{j}: x^{l} \geqslant 0\right\}, x=\left(x^{j} \in \mathbb{R}: j \in \mathbb{N}\right) \in l_{2}$.

Если $M$ компактно, то рассмотрим разбиения $Z_{k}$ с помощью подмногообразий с углами $M_{k}^{\prime}$ коразмерности 1 в $M$ такие, что $M=\bigcup_{j \in \Xi_{k}} M_{j, k}$, где $M_{j, k}$-подмногообразия, $\partial M_{j, k} \subset M_{k}^{\prime}$, $M_{i, k} \cap M_{j, k} \subset M_{k}^{\prime} \forall i \neq j, \lim _{k} \sup _{j} \operatorname{diam}\left(M_{j, k}\right)=0, \Xi_{k} \subset \mathbb{N}$. Пусть $H^{\gamma}\left(M, X ; Z_{k}\right)$ - пространства непрерьвных кусочно соболевских $H^{\gamma}$-функций [1] с нормой $\rho_{k, \gamma}(y):=$ $\left[\sum_{i \in \Xi_{k}}\left\|\left.y\right|_{M_{i, k}}\right\|_{H^{\gamma}\left(M_{i, k}, X\right)}^{2}\right]^{1 / 2}$ при $y \in H^{\gamma}\left(M, X ; Z_{k}\right)$ и $\rho_{k, \gamma}(y)=+\infty$ в противном случае, где $\infty>t \geqslant \gamma \in \mathbb{N}, \gamma \geqslant[m / 2]+1, m=\operatorname{dim} M$. Пусть $Y^{\gamma}(M, X)$ - это пополнение $\operatorname{str-ind~}_{j} H^{\gamma}\left(M, X ; Z_{j}\right)$ по норме $\|y\|_{\gamma}^{\prime}:=\inf _{k} \rho_{k, \gamma}(y)$, а для ограниченной области $W$ в $\mathbb{R}^{n}$ с кусочно $C^{\infty}$-границей пусть $Y^{\Upsilon, a}(W, X)$ - подпространство тех $f \in Y^{\infty}(W, X)$, для которых $\|f\|_{\Upsilon, a}:=\left(\sum_{j=0}^{\infty}\left(\|f\|_{j}^{*}\right)^{2} /(j !)^{a}\right)^{1 / 2}<\infty$, где $\left(\|f\|_{j}^{*}\right)^{2}:=\left(\|f\|_{j}^{\prime}\right)^{2}-\left(\|f\|_{j-1}^{\prime}\right)^{2}$ при $j \geqslant 1$ и $\|f\|_{0}^{*}=\|f\|_{0}^{\prime}, 0<a<\infty$. Если $W$ - это ограниченная область в $l_{2}$ с кусочно $C^{\infty}$-границей, а $H_{m}$ - подпространства, $H_{m} \subset H_{m+1}$ и $\operatorname{dim} H_{m}=n(m) \forall m \in \mathbb{N}, \operatorname{cl}\left(\bigcup_{m} H_{m}\right)=l_{2}$, то зададим $P_{\Upsilon, a}^{\infty}(W, X):=\operatorname{str}_{-i n d} Y^{\Upsilon, a}\left(W_{m}, X\right)$, где $W_{m}=W \cap H_{m}$, а $Y^{\xi}(W, X)$ определим

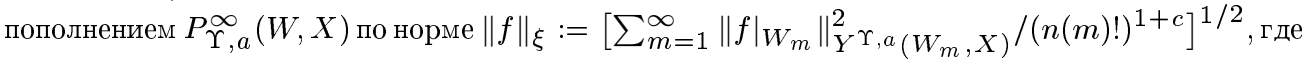
$0<c<\infty$ и $\xi=(\Upsilon, a, c)$. Пусть $M$ и $N$ являются $Y^{\Upsilon, a^{\prime}, c^{\prime}}$-многообразиями с $0<a^{\prime}<a$ и $0<c^{\prime}<c$. С помощью атласов $M$ и $N$ определим пространства $Y^{\xi}(M, N)$ и $Y^{\xi}\left(M, s_{0} ; N, y_{0}\right)$, где $\xi=(\Upsilon, a, c)$.

Если $M_{m}$ компактно и $f_{n, m} \in Y^{\xi}\left(M_{m}, N\right)$ имеет ранг $\operatorname{rang}\left(f_{n, m}(x)\right)=\operatorname{dim} T_{x} M_{m}$ $\forall x \in M_{m}$, то $f_{n, m}\left(M_{m}\right)$ является $Y^{\xi}$-подмногообразием в $N$ и на $f_{n, m}\left(M_{m}\right)$ зададим связность Леви-Чивита и риманов элемент объема $\nu_{n, m}$ такой, что $\nu_{n, m}\left(f_{n, m}\left(M_{m}\right)\right)=1$. Это индуцирует локальные нормальные координаты в $f_{n, m}\left(M_{m}\right)$. В случае $M_{m}=S^{1}$ получим параметр, соответствующий длине дуги на кривой. Если для $f \in Y^{\xi}(M, N)$ имеется последовательность $\left.f_{n, m(n)}\right|_{M_{m}} \in Y^{\xi}\left(M_{m}, N\right)$, сходящаяся к $f$, то получим $\psi_{f} \in Y^{\xi}(B, N)$ и $h_{f} \in Y^{\xi}(f(M), N)$ с $h_{f} \circ \psi_{f}=f$, где $B$ - это квадрант в $\mathbb{R}^{m}$ или $l_{2}$. В силу (i)-(iv) имеется вложение $\xi^{*}: Y^{\xi}(M \vee M, N) \hookrightarrow Y^{\xi}(M, N)$ [1], [2]. В сочетании с выбором натуральных координат получаем непрерывную композицию $g \circ f$ в $G:=Y^{\xi}\left(M, s_{0} ; N, y_{0}\right)$ такую, что 
$g \circ w_{0}=g$, где $w_{0}(M)=\left\{y_{0}\right\}$. Возьмем плотный подгруппоид $G^{\prime}:=Y^{\xi^{\prime}}\left(M, s_{0} ; N^{\prime}, y_{0}\right) \mathrm{c}$ $\xi^{\prime}=\left(\Upsilon, a^{\prime \prime}, c^{\prime \prime}\right)$, где $0<b^{\prime}<a^{\prime}<a^{\prime \prime}<a<\infty$ и $0<d^{\prime \prime}<c^{\prime}<c^{\prime \prime}<c<\infty, N^{\prime}-$ это $Y^{\Upsilon, b^{\prime}, d^{\prime \prime}}$-подмногообразие, плотное в $N$.

2. Теорема. На группоиде G существует квазиинвариантная бесконечно дифферениируемая мера $\mu$ относительно подгруппоида $G^{\prime}$.

Для доказательства рассмотрим $\varkappa \in Y^{\xi^{\prime}}(Q, M)$ так, что $\varkappa: \operatorname{Int}(Q \backslash S) \rightarrow M \backslash\left(A_{3} \cup \partial M\right)$ - гомеоморфизм, где $S$ - подмногообразие с углами коразмерности 1 в $Q, Q$ - квадрант в $X$. Для $M_{m}$ существует отображение $K(F, w)(v):=\int_{0}^{\zeta^{1}} \cdots \int_{0}^{\zeta^{m}}\left(F^{*} w\right) \quad \forall 0<a \leqslant a^{\prime \prime}<\infty$, где $B^{k} N=\bigoplus_{j=0}^{k} \Lambda^{j} N, \Lambda^{j} N$ - пространство дифференциалшных $j$-форм $w$ на $N, v=\varkappa(\zeta)$, $Q=[0,1]^{m}, N^{s}=N_{1} \times \cdots \times N_{s}$ с $N_{j}=N \forall j=1, \ldots, s$, причем $s \geqslant \operatorname{dim} M_{m} / \operatorname{dim} N$. Для последовательности гилшбертовых пространств $P_{q}$ над $\mathbb{R}$ с $q \in J \subset \mathbb{N}$ пусть $l_{2, \delta}\left(\left\{P_{q}: q \in\right.\right.$ $J\}):=\left\{x=\left(x^{q}: x^{q} \in P_{q}, q \in J\right) ;\|x\|_{l_{2, \delta}\left(\left\{P_{q}: q \in J\right\}\right)}:=\left(\sum_{q \in J}\left\|q^{\delta} x^{q}\right\|^{2} P_{q}\right)^{1 / 2}<\infty\right\}$, где $\infty>\delta \geqslant 0$. Возьмем $P_{q}=T_{w_{0}}\left(Y^{\xi} B^{k} M\right) \times \mathbb{R}^{l d} \forall q$, где $J=\mathbb{N}$ при $\operatorname{dim} N=\infty, J=\{1\}$ при $\operatorname{dim} N<\infty$. Пусть $e_{q} \in l_{2}\left(\left\{P_{q}: q \in J\right\}\right) \forall q \in J$ такие, что $\pi_{q}: P_{q} \hookrightarrow l_{2}\left(\left\{P_{q}: q \in J\right\}\right)-$ вложения с $e_{q} \in \pi_{q}\left(P_{q}\right)$ и $\left\|e_{q}\right\|_{l_{2}\left(\left\{P_{q}: q \in J\right\}\right)}=1$.

Если $\operatorname{dim} N=\infty$, то зададим отображение $K_{\infty}(F, w):=\left\{K_{m}\left(\left.F\right|_{M_{m}},\left.\omega\right|_{M_{m}}\right): m \in \mathbb{N}\right\}$, где $K_{m}$ определены для $M_{m}, \tilde{N}=N$ при $\operatorname{dim} N=\infty$ и $\widetilde{N}$ - подмногообразие в $N^{\infty}:=\bigotimes_{j=1}^{\infty} N_{j}$, моделируемое на $l_{2, d^{\prime}}\left(\left\{S_{j}: j \in \mathbb{N}\right\}\right)$ с $N_{j}=N \quad \forall j$, где $0<t-a<\left(a-a^{\prime \prime}\right) / 2$ и $0<q-c<\left(c-c^{\prime \prime}\right) / 2$, то получим отображение $K_{\Sigma}(F, w):=\sum_{m=1}^{\infty} \chi_{m}\left(K_{m}\left(\left.F\right|_{M_{m}},\left.w\right|_{M_{m}}\right)\right)$, где $\chi_{m}: Y^{\xi}\left(M_{n}, B^{n(m)} M_{m}\right) \hookrightarrow Y^{\xi}\left(M, B^{\infty} M\right)-$ вложения. Пусть $z=\left(z^{m} \in \mathbb{R}^{d(m)}\right.$ : $m \in \mathbb{N})$, где $z^{m}=\left.K_{m}\left(\left.F\right|_{M_{m}},\left.w\right|_{M_{m}}\right)\right|_{v=\varkappa(\zeta), \zeta=(1, \ldots, 1)}, d(m)=\operatorname{dim} B_{s_{0, m}}^{n(m)} M_{m}, z^{m}=$ $\left(z_{m}^{j}: j=1, \ldots, d(m)\right)$. Тогда $\|z\|_{q}^{2}:=\sum_{m=1}^{\infty}\left(\left\|z^{m}\right\|_{m}^{2} /(n(m) !)^{1+q}\right)<\infty$, где $\|z\|_{m}^{2}:=$ $\sum_{j=1}^{d(m)}\left|z_{m}^{j}\right|^{2}$; гильбертово пространство таких последовательностей обозначим $\bar{N}_{q}$. Тогда $K_{\Sigma}$ и экспоненциальное отображение $E$ многообразия $Y^{\xi}(M, N)$ порождают отображение $\bar{K}: W_{e} \times V_{e} \rightarrow V_{0}^{\prime}$, где $V_{0}^{\prime}-$ окрестность нуля в гильбертовом пространстве $H_{t, q}:=$ $\underline{T}_{w_{0}} Y^{\Upsilon, t, q}\left(M, s_{0} ; B^{\infty} M, s_{0} \times 0\right) \times \bar{N}_{q}$. Пусть $\Psi_{l, M, N}(f, w):=\Psi_{1, M, M}\left(\Psi_{l-1, M, N}\left(\mathrm{id}_{M}\right.\right.$, $\bar{K}(f,\langle w\rangle)))$, где $\Psi_{1, M, N}(f, w):=\bar{K}(f, w), l=2$ при $\operatorname{dim} M>1$ и $l=3$ при $\operatorname{dim} M=1$. Возьмем $P_{q}=H_{t, q} \forall q \in \mathbb{N}$. Существует семейство $\left\{w^{i, q, m}: i=1, \ldots, d(m) ; m \in \mathbb{N}\right.$; $q \in \mathbb{N}\} \subset T_{w_{0}} Y^{\Upsilon, \beta^{\prime \prime}, \gamma^{\prime \prime}}\left(M, s_{0} ; B^{\infty} \tilde{N}^{\prime}, y_{0} \times 0\right)$ для $a^{\prime}<\beta^{\prime \prime}<a^{\prime \prime}, c^{\prime}<\gamma^{\prime \prime}<c^{\prime \prime}$ такое, что отображение $\Phi(f):=\sum_{q=1}^{\infty} \sum_{m \in \mathbb{N}} \sum_{i=1}^{d(m)} \Psi_{l}\left(f, w^{i, q, m}\right) e_{q}$ инъективно, где $B^{\infty} \tilde{N}^{\prime}-$ это $Y^{\Upsilon, b^{\prime}, d^{\prime \prime}}$-подмногообразие в $\bigoplus_{j=0}^{\infty} \Lambda^{j} \tilde{N}^{\prime}, w^{i, q, m}$ независимы от локальных координат $\left(x^{n(m)+1}, x^{n(m)+2}, \ldots\right) \quad \forall i, q$ и $m$. Поэтому $\Phi-$ дифффеоморфизм окрестности $U_{0} \ni w_{0} \in$ $Y^{\xi}\left(M, s_{0} ; N, y_{0}\right)$ на окрестность $V_{0} \ni 0$ в гильбертовом подпространстве $K_{0}$ в $l_{2}\left(\left\{P_{q}: q \in J\right\}\right)$. Сушествуют окрестности $V^{\prime} \ni w_{0} \subset G^{\prime}$ и $U_{1} \ni w_{0} \subset G$ такие, что $V^{\prime} \circ U_{1} \subset U_{0}$. Пусть $S_{\varphi}(v):=\Phi \circ \eta \circ \Phi^{-1}(v)-v$ c $v \in V_{1}, \eta \in V^{\prime}$, где $V_{1}=\Phi\left(U_{1}\right)$. Поэтому гауссова мера на $K_{0}$ с помощью $\Phi$ индуцирует меру на $G$ (см. также [1], [2]).

\section{СПИСОК ЛИТЕРАТУРЫ}

[1] С. В. Людковский // Докл. РАН. 2000. Т. 370. № 3. С. 306-308. [2] S. V . Ludkovsky. Generalized Loop Groups of Complex Manifolds. Preprint math.RT/9910086. Los Alamos: Los Alamos Nat. Lab., 1999. 